\title{
Biorobotics:
}

\section{Using robots to emulate and investigate agile animal locomotion}

\author{
Auke J. Ijspeert
}

\section{Affiliation:}

Biorobotics Laboratory, EPFL - Ecole Polytechnique Fédérale de Lausanne, CH-1015 Lausanne, Switzerland. Auke.Ijspeert@epfl.ch

\begin{abstract}
:
The graceful and agile movements of animals are difficult to analyze and emulate because locomotion is the result of a complex interplay of many components: the central and peripheral nervous systems, the musculoskeletal system, and the environment. The goals of biorobotics are to take inspiration from biological principles to design robots that match the agility of animals, and to use robots as scientific tools to investigate animal adaptive behavior. Used as physical models, biorobots contribute to hypothesis testing in fields such as hydrodynamics, biomechanics, neuroscience, and prosthetics. Their use may contribute to the design of prosthetic devices that more closely take human locomotion principles into account.
\end{abstract}

\section{Main Text:}

A cat running, climbing, jumping, and rapidly catching moving objects is fascinating to watch. Performing these agile motor behaviors requires complex interactions among the central nervous system, the peripheral nervous system, the musculoskeletal system and the environment. Such good locomotion abilities are fundamental for animals, and also useful for robots. The field of biorobotics - the construction of biologically-inspired or biomimetic robots - takes inspiration from biological principles to design robots with sensorimotor skills that approach those of animals. This has led to fish-like (1-4), snake-like (5-7), cat-like (8-12), and humanoid robots (13-15) (Fig. 1) with possible applications in search-and-rescue, environmental monitoring, agriculture, transport, and construction.

Biorobotics is increasingly contributing back to biology in fields such as biomechanics and neuroscience. Indeed, biorobots are becoming important scientific tools $(4,16,17)$, and can be used to investigate locomotion and to test hypotheses about the underlying interactions of body, control, and environment. Robots have multiple properties to complement animal studies: Their actions are repeatable, they offer access to variables or quantities that would be difficult to measure on animals, they can perform movements that are unnatural or dangerous for animals, and their morphology can be systematically changed. Biorobots are providing useful contributions to biomechanics $(10,14,18)$, neural control of movement $(9,19)$, prosthetics $(20$, 21 ), and environmental interaction mechanics; such as hydrodynamics $(1,3)$, and the new field of terradynamics, the dynamics of sand and other granular media (22), that was established with the use of biorobots. 

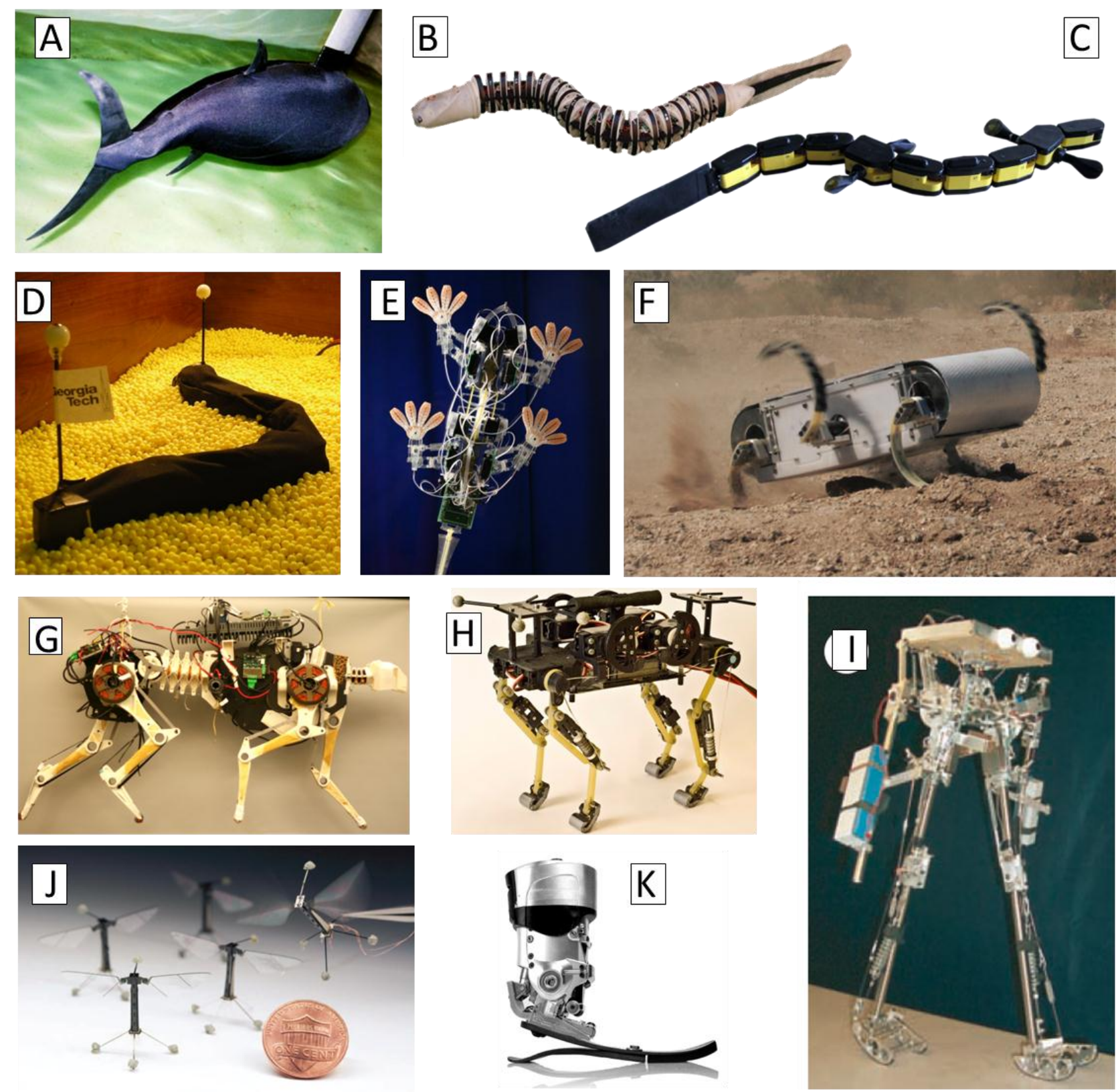

Fig. 1. Example of biorobots. (A) RoboTuna (35). (B) Lamprey robot (39). (C) Salamandra robotica (19, 67). (D) Sandfish lizard robot (6). (E) StickyBot (56). (F) RHex (22, 54). (G) MIT Cheetah robot (8). (H) CheetahCub (10). (I) Cornell biped (74). (J) Miniature flapping wing robot (46). (K) Powered ankle-foot prosthesis controlled by a neuromuscular model (20). Permissions: (A) M. Triantafyllou; (B) IOP Publishing; (C, E, G, K) IEEE; (D) The Royal Society; (F, I, J) AAAS; (H) A. Sproewitz.

As a first approximation, animal locomotion is based on two key principles: the generation of periodic movements using muscles (which is quite different from the rotational movement of electromagnetic motors), and the generation of asymmetries in the interaction forces with the environment, such that periodic movements of muscles are transformed into a forward acceleration (as opposed to back-and-forth movements in place). Depending on the ecological 
niche, nature has evolved a large variety of different morphologies and ways of generating these asymmetries: elongated bodies with traveling waves for swimming, scales in snakes that provide asymmetric friction for crawling, and limbs that alternate between (high-friction) stance and (low-friction) swing for walking $(23,24)$.

Although the underlying principles appear simple at first glance, understanding animal locomotion is complex because it is a problem that (i) involves complex dynamic interaction among many elements (multiple neurons, muscle fibers, bones, tissues, and all elements in the environment), (ii) is therefore high-dimensional, (iii) is highly nonlinear (e.g. doubling the contraction of a single muscle at a given time will not lead to a doubling of the locomotion speed), and (iv) is multi-disciplinary. The biomechanics of locomotion requires the investigation of all the internal forces in the high-dimensional musculoskeletal system, but also all the complex interaction forces with the (unstructured) environment, with the further complication that the interaction will change the environment itself such as displacement of water or sand.

Understanding locomotion therefore requires a systems-level approach that explores the interaction of all involved components $(23,25)$, in addition to studying components in isolation. Such an approach comes naturally in robotics, which is by essence the science of integration of many components (materials, actuators, sensors, and control loops). Biorobots can play a key role in animal locomotion studies thanks to an "understanding by building" approach (26). Complementing other reviews in biorobotics $(12,15-17,25-29)$, the focus here will be on locomotion, on vertebrate animals, and on the use of robots as scientific tools to explore the biology of locomotion.

\section{Swimming}

Swimming involves complex interactions between a deformable body (e.g. a fish undulating its body and/or flapping its fins) and water motion. The interaction forces generate complex water displacements, and can lead to surprising behavior, such as a dead trout swimming upstream when placed downstream of a fixed cylinder (30), or Gray's paradox: Using an estimated drag coefficient of a rigid body, Gray concluded that the ratio between drag power and muscle power appeared too large by almost an order of magnitude for a dolphin to reach its observed swimming speed $(31,32)$. It is now known that fish swimming strongly depends on the interaction of the body with vortices (i.e. spinning motion of water), in particular periodic patterns of vortices called Karman streets, and that fish "exert precise and effective control of the flow around their bodies to extract energy from waves, turbulence and even their own wakes" (32). Gray's paradox therefore appears to be due to an overestimation of the drag because of the rigid body assumption as well as an underestimation of the peak muscle power of dolphins (32, $31)$.

Biorobotics can play an important role in exploring the underlying physical phenomena, and in testing hypotheses about the mechanisms of fish swimming $(1,4,33)$. It can also benefit from the impressive swimming skills of fishes in terms of agility [e.g. the ability to rapidly turn without losing much speed (32)] and energy efficiency [e.g. the ability of eels to swim thousands of kilometers with little or no food (34)]. Different types of robotic devices have been used in these studies: (i) robotic devices with actuated fins that are attached to a fixed or (externally) moving basis, typically in a flow tank (32); (ii) robotic devices that are self-propelled while 
being attached to a low-friction rail (Fig. 1A) $(1,35)$; or (iii) freely moving fish-like robots $(2-4$, $36-40)$.

A self-propelled robotic pectoral fin was used in studies of the interaction between deformable fins and water (1). Three different fin motions were compared, while recording interaction forces with water at the base of the fin. One of the motions, 'cup and sweep', closely resembled the motion of a sunfish and led to the highest thrust forces. The forces closely match predicted force patterns from a computational fluid dynamics simulation based on the actual movements of the sunfish pectoral fins (41) and were in agreement with particle image velocimetry analyses of water flow around the fish (1). The authors also designed a device made of two foils to investigate the interaction between dorsal and anal fins (first foil) and the caudal fin (second foil). It was shown that the interaction of two foils can be beneficial for thrust enhancement, with the first foil shedding a distinct vortex wake that markedly alters incoming flow to the second foil and causes increased leading edge suction, in agreement with predictions from a computational study (42).

Some fishes, like the glass knifefish, perform fin movements that generate forces in other directions than necessary for forward locomotion, sometimes even against it, which looks like a waste of energy (2). The hypothesis is that these movements are performed to enhance the control of locomotion, and to reduce the trade-off between stability (e.g. the ability to keep a steady speed and heading) and maneuverability (the ability to accelerate and turn) that animals and robots face. The glass knifefish uses a single elongated ventral fin to hover and rapidly change direction (Fig. 2A). The fin performs undulatory movements in opposite directions along different parts of the body, typically with two inward-traveling waves that meet at a nodal point (Fig. 2C). It was observed that the position of the nodal point was modified depending on the speed of swimming, moving towards the tail for higher forward speed. This was then tested using a mathematical model and a robot (Fig. 2B), and it was found that the resulting thrust force varied linearly with the shift of the nodal point. It was also found that the counteracting waves lead to a passive damping effect that helps rejecting perturbations of swimming velocities (that could be due to perturbations of the water around the robot), and that can be adjusted by the frequency of the undulation. Together these two mechanisms nicely enhance both the maneuverability (by shifting the nodal point) and stability (by adjusting the damping) of glass knifefish swimming relative to a swimming mode that uses a single wave along the whole fin with changes of frequency for changing direction. The increase of maneuverability is especially striking for small-amplitude movements. 



Fig. 2. A biomimetic robot that emulates the hovering performance of the glass knifefish (2). (A and B) To mimic the kinematics of the glass knifefish (A), the motorized fin was programmed to produce two inward-traveling waves that meet at a nodal point (B). (C) Experiments with the fish and robot were explained by a computational model. By shifting the nodal point and altering the frequency of the undulation, both the direction of thrust and the damping of perturbations can be adjusted, offering a way to simultaneously adjust maneuverability and stability respectively. Permission: N. Cowan.

\section{Flying}

There is currently a boom in flying robots, in particular robots with rotating wings such as quadropters and fixed-wing robots (43). Self-propelled flapping-wing robots, also called ornithopters, are less common (44). They range from miniature robots (45-47) to the SmartBird by Festo (a flying robot inspired by the herring gull), toys (such as a flying pigeon-like robot by E-Bird and the Flytech Dragonfly by WowWee), and large-scale ornithopters capable of carrying a person (48). 
Flapping-wing robots have been very useful as physical models to investigate insect and bird flying. Similarly to Gray's paradox for swimming, the wings of insects appear unable to generate sufficient lift when maintained statically in air flows at constant velocities (in the same range of velocities as those of flapping) (49), which suggests that the flapping and rotational movements of the wings as well as the vortices shed by the wings are important to generate the lift forces necessary for flight (50). Taking advantage of the similarities of fluid dynamics in air and in water, studies of the physics of insect flapping wings have used dynamically scaled robotic wings in mineral oil (i.e. with adjusted dimensions, frequencies, and oil viscosity to match the Reynolds number of the insect flight) (50). By equipping the actuated wings with force sensors, it was possible to investigate how lift can be generated by flapping wings, and it was found that insect flight could be explained by the interaction of three mechanisms: delayed stall (during stroke), rotational circulation, and wake capture during stroke reversal (50). The mechanisms of wake capture in which a wing benefits from the vortices generated by the previous stroke, are similar to those found with fish robots (42). Furthermore, by simply modifying the timing of the rotational movements, the direction of forces can be adjusted and hence the direction of flying can be modulated, both for left-right yawing and up-down movements. These changes of timing resemble the changes of movements observed during steering behaviors in Drosophila (50). Similarly, it was found that yaw movements can be obtained with small adjustments of the stroke plane angle and the stroke amplitude (51), and that forward speed could be regulated by wing movements that alter pitch (52). Like the findings of the glassfish study described above, propulsion and steering are therefore closely merged and obtained by subtle modulations of propulsive movements; this is quite different from most fixedwing and propeller-based underwater robots where some motors are dedicated for propulsion and others for steering (e.g. using rudders).

Although flying based on flapping-wings is currently outperformed by propeller-based flying for robots of the weight of birds or more, it is well-suited for robots of the size and weight of insects. An impressive miniature 80-milligram flapping wing robot has been designed $(45,46)$ (Fig. 1J). The authors note that "conventional technologies for macroscale aircraft propulsion and manufacturing are not viable for millimeter-scale robots because of inefficiencies that arise from force scaling, suggesting a biologically inspired solution based on flapping wings". The robot could exhibit stable hovering and simple flight maneuvers. The rotational motion of the wings was obtained by combining active flapping with passive pitch rotation thanks to passive compliant flexures. The resulting movements resembles those described above (50) and similarly generate sufficient lift forces for flight. For future work, the authors note that such robots could be used to study the mechanics and control of insect flying, and may enable the measurement of forces and torques during free flight that could be difficult to simulate in scaled models.

\section{Crawling and terradynamics}

Many animals locomote on granular media such as dry sand or gravel. Granular media are complex media that can exhibit both solid-like and fluid-like features $(22,53)$. Some animals can even swim through sand; the sandfish lizard uses a large-amplitude traveling wave (53) not unlike the swimming of water snakes. The modeling of locomotion in granular media has led to the new field of terradynamics in which robots play a big role $(6,22)$. More generally a range of snake robots have been constructed that, like their biological counterparts, perform motion through multiple contacts with the environment $(5,7)$. 
High-speed x-ray imaging revealed that the lizard swims in sand by means of body undulations without the help of limbs. Making the hypothesis that the animal swims in a socalled "frictional fluid" in which grain-grain and grain-animal friction determine drag and thrust forces, the authors developed an empirical model of sand swimming that shares similarities with swimming in liquids with low Reynolds-numbers (e.g. with negligible inertia effects), but with a mechanism for drag that is frictional (i.e. velocity-independent) instead of viscous (53). The model showed good agreement with the experiments and could predict the wave efficiency and optimal kinematics. A robot model of the lateral undulations of the lizard was later developed to further validate the model (Fig. 1D) (6). The robot was useful for systematic testing of different types of body undulations, in particular with different ratios between amplitude and wavelength. The robot proved to be a good match with the empirical model. Interestingly, they both obtained maximal speeds when the ratio between amplitude and wavelength was 0.2 , the same as that used by the sandfish.

The same group extended their terradynamics model to predict interaction forces induced by arbitrarily-shaped legs and bodies moving freely in granular media (22). A RHex-like robot (54) with six rotary legs was tested with different types of leg shapes in different types of granular media (Fig. 1F). The authors obtained a remarkable match between experimental data and the model, for instance in terms of the interaction forces of rotating legs of different shapes with the granular media, and in terms of locomotion speeds of the robot with different leg shapes and different stride frequencies. Such a terradynamics model can be useful to understand how lizards run in the sand (55) and to design leg shapes and control laws for robots that move in sand and gravel.

\section{Climbing}

Climbing has also been studied in robots (56-60), in particular the impressive climbing abilities of gecko lizards that exhibit directional dry adhesion under their feet (61). On the basis of an analysis of the feet and the small hairs that provide directional adhesive to the gecko, the toes of a lizard-like robot (Fig. 1E) were equipped with arrays of small-angled polymer hair manufactured using shape deposition (50). Despite being at least two orders of magnitude larger than gecko hairs, this led to similar directional adhesion that was sufficient to carry the robot on vertical surfaces of glass or other smooth surfaces. Together with the compliance of the feet, the robotic toes adhere to a surface when pulled toward the ankle, and are easily released when pulled in the other direction. Relative to non-directional adhesives, the foot required much less pulling force to detach from the surface. This explains the ease and rapidity with which geckos can climb on walls and ceilings (61). For proper climbing, the robot required movements and postures such that feet are always pulled towards each other-something that has been also observed in geckos that reorient their feet as they climb in different directions.

\section{Quadruped walking and running}

A range of quadruped $(8-12,62)$ and multi-legged robots $(54,63)$ have been constructed to date, including robots developed by companies for which no scientific reports exist (e.g. BigDog and WildCat by Boston Dynamics); see $(12,15)$ for reviews. Many of these were designed to emulate the walking and running skills of tetrapods, such as climbing over complex 
uneven terrain and crossing terrain with limited footholds (e.g., stones in a river); they benefit from the advantages offered by discrete contacts with the ground versus continuous contacts through wheels or tracks,

One drawback of many legged robots is their low energy efficiency, as illustrated for instance by their large costs of transport (CoT, the ratio of power consumption to the product of weight and speed), a dimension-less measure of energy efficiency for locomotion (8). Several principles for reducing these energy costs were proposed by $\operatorname{Kim}$ and colleagues $(8,18)$ : the "employment of high torque density motors, low impedance transmission, energy regenerative electronics and a design architecture that minimizes the leg inertia". The last principle has led the authors to design a lightweight leg that followed the hypothesis that vertebrate legs are organized such that bones carry only compressive loads while the muscles, tendons and ligaments carry tensile loads, in order to reduce bending torques (64). The robotic legs were constructed using Kevlar cables for tendons and lightweight bone-like structures made of foam-core composite fabrication (Fig. 1G). By using the principle of tendon-bone co-location, stress on the bone during a stride could be reduced by up to $59 \%$ relative to a leg configuration without tendon (18). Combined with actuators that have low gear ratios (and therefore low friction) and electronics that allow recapture of energy when the motor brakes, the robot is capable of fast locomotion $(2.51 \mathrm{~m} / \mathrm{s})$ at a CoT of 0.51 ; according to the authors this is significantly lower than that of BigDog (estimated CoT of 15) and is comparable to running animals at the same scale.

In related work, it was shown that replicating the pantograph-like structure of a mammal limb and approximating its viscoelastic properties (Fig. 1H) can lead to surprisingly robust and dynamics gaits purely with open-loop control (10). Relatively big perturbations such as walking down a step did not require sensory feedback nor complex closed-loop control but were in fact dampened-out by the mechanical properties of the robot. Similar mechanical self-stabilization mechanisms have been identified in running cockroaches (65).

Quadruped robots have also been used for testing hypotheses related to the neural control of motion, for instance in the cat (9) and the salamander (19). The salamander uses an anguilliform swimming gait in water and a walking trot gait on the ground. The locomotor patterns are generated by neural circuits in the spinal cord called central pattern generators. Gait transitions between the two modes of locomotion can be induced in a decerebrated animal by electrical stimulation of a region in the brainstem, with walking-like patterns at low stimulation, and swimming-like patterns at high stimulation (66). This illustrates that spinal cord circuits not only can produce well-coordinated movements but can even generate gait transitions under simple descending control signals. A salamander-like robot (Fig. 1C, 3A) was used to test the hypothesis that the salamander central pattern generator is based on an ancestral lamprey-like swimming neural circuit for its axial musculature extended during evolution by specialized and slower neural oscillators for the limbs (19). The model and the robot could replicate the gait transition induced by electrical stimulation. It also provided an explanation of why walking gaits (Fig. 3B) are systematically performed at lower frequencies than swimming gaits (Fig. 3C) in the animal. Finally the robot demonstrated that the particular body-limb coordination used by salamander on the ground is the one among several options that optimizes its locomotion speed (67). 



Fig. 3. Salamandra robotica, a salamander robot that can swim and walk, was designed to test hypotheses about the organization of salamander spinal circuits and the mechanisms of gait transition $(19,67)$. (A) The water-proof robot is equipped with eight motors for spine undulations, and four motors, one per leg, for leg rotation. (B) Comparison of the walking trot gait of the robot (left) and the salamander, as recorded with x-ray videos (right). (C) Comparison of the swimming gait of the robot (left) and the salamander (right) (67).

The mechanisms of inter-limb coordination, and in particular the respective role of neural coupling versus mechanical coupling, have also been investigated using a quadruped robot (68). It was shown that stable gaits could be generated without direct coupling between limb oscillators, and with only indirect coupling through sensory feedback and mechanical coupling, similar to what has been observed in the stick insect (69). The robot was a useful tool to demonstrate that different gaits could be obtained depending on the mass distribution in the robot. When the mass was placed more in the front as in camels or more to the rear as in monkeys, the same gaits emerged as in their biological counterparts.

\section{Biped locomotion}

Two broad classes of biped and humanoid robots can be distinguished: (i) robots that are designed to be versatile, and (ii) passive-dynamic robots that are designed to be energy-efficient. Versatile robots use multiple high-torque actuators and sophisticated control algorithms to carefully control all joints at any given time. This has led to impressive machines such as Asimo (Honda), Qrio (SONY), Atlas (Boston Dynamics), Shaft's biped, and HRP (AIST and Kawada 
Industries); see (15) for a review. However, from a biomechanical point of view, these robots are far from human-like because they require actuation to perform any motion, as opposed to human walking that relies extensively on natural dynamics of the musculoskeletal system (such as pendulum-like swinging movements of the limb). Such robots are therefore highly inefficient from an energy point of view.

Passive-dynamic walking robots are more human-like in terms of biomechanical aspects and energetics, and are interesting tools for exploring the biomechanics of human locomotion $(13,14,70)$. This type of locomotion is called passive because it relies on passive dynamical properties of the body such as free swinging motions (as opposed to motions that are actuated at all times), and dynamic because it is dynamically stable (i.e. a notion of stability over time) as opposed to statically stable (i.e. with the center of gravity remaining at any time over the support polygons shaped by the contact points of the feet on the ground). Note that most versatile robots also perform dynamic locomotion.

Inspired by ramp-walking toys and abstracting walking as a wheel without a rim, McGeer (14) using nonlinear stability analysis and by building prototypes demonstrated how a passivedynamic walking machine could be constructed to get down a ramp without actuation and control. With a well-tuned body morphology made of two straight legs with round feet, swinging motions of the limbs and stable walking could be obtained thanks to gravity and inertia alone, without the need for careful control of limb motions. This led to a paradigm shift in biped locomotion, going away from trajectory-based control towards locomotion that is tightly based on passive properties of the body, much like humans are believed to do (71-73).

For instance, a more human-like 3D passive-dynamic walker with knees and counterswinging arms produced strikingly human-like features (13). Subsequent developments included the addition of actuation to remove the necessity of a ramp, and of learning algorithms to learn suitable control policies online, i.e. while walking (74). This has led to three robots (the Cornell, Delft, and MIT bipeds) that "use less control and less energy than other powered robots, yet walk more naturally, further suggesting the importance of passive-dynamics in human locomotion" (74). The Cornell biped (Fig. 1I) was designed to minimize energy loss that happens in human and robot walking when the foot hits the ground and when actuators actively brake movements and perform negative work. By completely avoiding negative work, the robot could walk with a low CoT of $\sim 0.2$, which is equivalent to human walking (CoT of 0.2) and is an order of magnitude lower than Honda's Asimo (estimated CoT of 3.2). Further the locomotion of the robots required very little control effort relative to trajectory-based control. The Cornell and Delft bipeds used very simple control laws directly linking ground contact sensors to on/off motor commands sent once per step. The MIT biped used online reinforcement learning to optimize a control policy during locomotion. Because the intrinsic mechanical stability simplified the learning problem, the learning was sufficiently rapid that the robot could continuously adapt to the terrain during walking. This work showed that with the right mechanics, human locomotion is energy-efficient and possibly less difficult to control than originally thought. The passive-dynamic walking robot Ranger (1 meter high, $9.9 \mathrm{~kg}$ ) could walk $65 \mathrm{~km}$ over 31 hours on a single battery charge $(\sim 500 \mathrm{~W} \cdot$ hours, CoT of 0.28$)$ —an impressive feat (75).

The interaction between passive-dynamic walking and neuronal control with simulated synaptic plasticity has been further explored (76). It was shown that a robot maintained in the sagittal plane could walk with high speed and could learn to walk on different terrains with only 
a few learning iterations. The authors concluded that "the tight coupling of physical with neuronal control, guided by sensory feedback from the walking pattern itself, combined with synaptic learning may be a way forward to better understand and solve coordination problems in other complex motor tasks" (76).

The versatile and passive-dynamic approaches can to some extent be merged in robots that exploit torque-control in addition to position-control (77-81). Such robots typically use whole-body model-based control together with optimization algorithms. By taking into account natural dynamics in the dynamic model of the robot, and by adding energy criteria in the optimization it is in principle possible to generate locomotion that is both versatile and energyefficient. Torque-controlled robots also offer the opportunity to easily test biomechanical hypotheses without having to rebuild all the mechanics (82). They can emulate muscle models using active impedance, while the real physics is still taking care of the part that is difficult to simulate part, i.e. the contacts and interactions with the environment.

\section{Active exoskeletons and prostheses}

Active exoskeletons (for limb support) and prostheses (for limb replacement) are fields in which robotics, biomechanics, and human motor control converge (83). In order to restore or augment human locomotion a series of actuated exoskeletons have been designed, including BLEEX (84), HAL (85), Sarcos' exoskeleton, MIT Exoskeleton (86), MINDWALKER (87), and RoboKnee $(88)$; see $(83,89)$ for reviews. Several can be bought as commercial products, such as the ReWalk from ReWalk Robotics and Ekso from Ekso Bionics. Potential users are soldiers, workers, or persons with a locomotor handicap (for instance due to a spinal cord lesion).

To reduce the size and weight of fully actuated exoskeletons, researchers have investigated the energetics of human locomotion. Closely linked to the passive-dynamics walking studies mentioned above, numerical models and optimization have shown that purely passive exoskeletons made of elastic tendons should in principle be capable of reducing the metabolic cost of locomotion for specific movements (90). Such ideas have led to the design of exoskeletons for helping to carry load with little actuation-for instance in an exoskeleton equipped with springs at the hip and ankle, and a variable damper at the knee (86).

Going even further, it is possible to harvest energy from human walking. One examples is the use of a knee brace that collects energy at specific movements during the walking cycle, during late swing when knee muscles normally perform negative work to prevent hitting the joint-angle limit (91). Such a device can produce an average of 5 watts of electricity, with little extra effort by the wearer. Alternative methods include harvesting the energy lost in shoe soles at impacts with the ground (92) or in periodic movements of backpacks (93).

Similarly to exoskeletons, powered prostheses for ankles and knees have been developed and have become commercial products from companies such as Ottobock, Össur, and SpringActive. An interesting approach used a simulated neuromuscular model to drive a powered ankle-foot prosthesis (Fig. 1K) (20). The controller was based on a simulated Hill-type muscle with a positive force feedback reflex, replicating the reflexive muscle response based on feedback signals from muscle spindles and Golgi tendon organs in human walking (94). Compared to other approaches that play a fixed torque pattern, the motivation is to adjust the torque produced by the prosthesis depending on the slope of the terrain and the size of steps. The 
system was tested with a transtibial amputee walking on level ground, and up and down a ramp. It was found that the energy provided by the prosthesis was adapted to the type of terrain and was directly correlated to the ground slope angle. Also the gait characteristics were close to those of intact locomotion in terms of the measured ankle torque and ankle angle profiles. In subsequent work, it was found that the approach could successfully be used for speed adaptation (95) and that it decreases metabolic cost by $8 \%$ and increases preferred walking speed by $23 \%$ relative to using a passive-elastic prosthesis (21).

\section{Conclusion, future prospects and implications.}

Biorobotics is an exciting research area with two main objectives: (i) taking inspiration from biological principles to design robots that match the agility of animals, and (ii) using robots as scientific tools to investigate animal adaptive behavior.

Although the two objectives share many common aspects and methods, they also differ in some subtle but important points. First, the evaluation of success is different. A contribution to robotics (i.e. to the first objective) will be considered successful if it provides a method that is better (according to some performance metric such as speed or energy efficiency of locomotion) or simpler to implement than alternative methods. The RHex robot (54) is a nice example of such a contribution. A contribution to biology (second objective) will be considered successful if it contributes to a scientific theory either by formulating new hypotheses, proposing new experimental methods, validating experiments against animal data, and/or providing new theories (e.g. mathematical formulation) - for instance, the studies of terradynamics of the sandfish lizard $(6,53)$ and of the tradeoff between stability and maneuverability in the glassfish (2). It is important for a project to properly define which of the two (or sometimes both) objectives it is aiming at. Otherwise there is the risk that a project does not contribute to robotics (because it does not outperform other methods) nor to biology (because it does not satisfy scientific standards of well-established hypotheses, methods, and experiments).

A second important point is the choice of the level of abstraction and which features of an animal should be replicated in the robot. Researchers are mostly aware that animal locomotion is not "optimal" in any sense, but just "good enough" from an evolutionary perspective, and that animals and evolution need to satisfy many constraints such as growth, reproduction, metabolism, etc. , that are not relevant for robot locomotion. Therefore it is important to only replicate the key relevant features. For instance, a multi-segmented leg can be approximated with a rotary leg driven by a single rotational motor $(19,54,96)$, and a simple wheeled robot with an active tail can be a useful tool to investigate active tail stabilization in lizards (97). To formalize the choice in the level of abstraction, Full and colleagues introduced the useful concepts of templates and anchors $(25,98)$. A template is "the simplest model (least number of variables and parameters) that exhibits a targeted behavior" (98). For instance the spring-loaded inverted pendulum model of running (99) is a template. An anchor is a more elaborate model that takes into account many more aspects of the musculoskeletal and the nervous systems (e.g. with multisegmented legs, multiple muscles, and multi-layered neural control loops). Depending on the type of scientific questions, the right level of abstraction between template and anchor should be carefully chosen [see $(16,25)$ for in-depth discussions]. 


\section{From robotics to biology}

Biorobots can give something back to biology. Like numerical models that are now routinely used in any field of science, robots can become part of the scientific cycle of making hypotheses and predictions, testing them in experiments, and iteratively adjusting them towards a scientific theory (16).

Robots can be programmed to make repeatable, parameterized experiments (1). They can be equipped with multiple sensors to monitor relevant variables/quantities that would be difficult to measure on an animal [e.g. internal forces, see $(11,62)]$. Their morphology can be modified in systematic ways (22). They can perform movements that would be dangerous for animals. They can perform movements that animals typically not do $(1,67)$, allowing one to explore whether animal movements are optimizing some criteria [see the salamander study that showed that the walking trot gait of salamander optimizes speed (67)]. Given today's computer and communication technologies, they can be controlled by numerical models (controllers) that replicate some parts of the central nervous system in great detail. With the right type of actuators and materials, they can properly approximate visco-elastic properties of the musculo-skeletal system [e.g. with virtual muscles and torque-controlled motors (82), or with muscle-like actuation (70)]. In short, robots can be used to perform some experiments that would be difficult or even impossible to make with animals. In the long run, they could even reduce the need for some types of animal experimentation.

Webb extensively discussed the use of robots as physical models and proposed seven dimensions (relevance, level, generality, abstraction, structural accuracy, performance match, and medium) to characterize and compare models (16). As pointed out by Long, the classification is not perfect (e.g. the dimensions are rather qualitative and lack scales) but is very useful to challenge "researchers to explicitly justify their use of robots to make and test biological hypotheses" (4).

\section{Why use robots and not simply numerical simulation?}

Robots and numerical simulations are complementary, and in many cases numerical simulations precede robotic experiments $(6,19)$. There is a long history of mathematical and numerical models of animal locomotion (25); biped locomotion has been modeled using simple abstract models (i.e. templates) like the spring-loaded inverted pendulum model (99) as well as by complex neuromechanical (anchor) models (94).

Robots are better than numerical simulations when real (as opposed to simulated) physics is important. This is often the case with locomotion that involves complex physical interactions with the environment: eswimming in water (1-3), crawling on sand (6), and walking on mud or gravel. Mechanical simulations are now very good at computing the dynamics of articulated rigid bodies but are less good at correctly simulating compliant structures and interaction forces with a complex environment. Such problems are very complex for various reasons: They involve complex geometries, complex physics, deformations of whole structures, multiple materials, non-stationary phenomena with often abrupt changes, and induced changes in the environment (e.g. motion of water, sand, or gravel). For instance consider a salamander robot that swims in water in contact with a rocky ground, then crosses mud and climbs over a grassy terrain. Properly formulating all the underlying equations of motion and boundary conditions is 
tremendously hard. Finally, as visible from the many impressive robots designed by hobbyists, constructing a robot allows for tinkering-itself a powerful iterative design process based on intuition that can lead to impressive constructions and that is quite different from creating a numerical model on a computer (13).

There are however a number of difficulties in using robots for investigating animal locomotion that should not be underestimated [as discussed in (29) and briefly reported here]. It is difficult to properly replicate the biomechanical properties of animal musculoskeletal system (e.g. the same number of degrees of freedom, the viscoelastic properties, and the mass distribution). There is therefore a tradeoff between the benefits of real physics and the risk of designing a robot that has significantly different dynamics from that of the modeled animal. This relates to choosing the right level of abstraction between anchors and templates $(25,98)$. Also some biological sensor modalities such as touch and proprioception are still difficult to properly replicate with current sensor technology. Finally, using robots is sometimes more cumbersome than using numerical simulation because robots are less adjustable, require a large overhead and expertise for construction and maintenance, and are less amenable to extensive experiments.

\section{Future challenges and opportunities}

There remain multiple challenges that roboticists and biologists can tackle together. Some of the main challenges include designing and controlling robots that are really field-ready, designing robots capable of multimodal locomotion, studying non-steady-state behavior, and quantifying and achieving agility.

Apart from a few exceptions [e.g. $(7,67)$ and robots from BostonDynamics such as RHex $(54)$ and BigDog], most biorobots are not yet field-ready; they are not sturdy enough to resist water, dust, mud, and falls, and cannot adapt their locomotion to complex environments. A lot of scientific and engineering work is still needed to make robots field-ready, not only in terms of mechanics and actuation [e.g. with muscle-like variable impedance actuators $(70,100)$ ], but also in terms of integrating multimodal sensing for perceiving unstructured terrain (e.g. identifying the right footholds for walking and climbing), adding better proprio- and tactile information (e.g. to identify when stuck), and improving control. An interesting approach towards sturdiness and more animal-like mechanical properties comes from the rising field of soft-robotics [see (101) for a review], with the design of robust robots that can move in multiple terrains and survive harsh conditions (102). Progress towards outdoor tests will not only help to make robots more useful for applications in search-and-rescue, environmental monitoring, agriculture, transport, and construction, but will also help (and be guided by) research on animal locomotion in their natural ecological niches.

Unlike their biological counterparts (103) few robots are capable of multimodal locomotion, the ability to switch between different gaits and motor behaviors (e.g. a cat running, jumping, crawling, climbing, standing up, etc.). Exceptions include RHex (and AQUA its aquatic version) that can walk and swim $(54,104)$, and the salamander robot than can swim, crawl, and walk (19). For many outdoor applications, the ability to perform multimodal locomotion will be important for crossing many types of environments without getting stuck. Interesting scientific questions remain to be addressed with robots to better understand the interplay (and necessary trade-offs) of morphology, energetics, and control in multimodal locomotion. 
Studying non-steady state behavior is difficult. Except possibly for flying $(50,51)$, most studies in biology and in robotics have so far focused on steady-state behavior, e.g. analyzing periodic gaits of straight-line locomotion at a given speed. The main reason for this is that making experiments and collecting data for steady-state behavior is much easier than for nonsteady-state behavior. But freely-moving animals are rarely in steady-state: they continuously accelerate/decelerate, turn, switch between gaits and different motor behaviors, superimpose motor behaviors, etc. From a dynamical systems point of view, they are mostly in transient mode rather than in steady-state. Biorobots being more amenable to experiments than animals can become key tools to study non-steady state locomotion.

Related to this, there is still a lack of quantitative metrics to measure agility, both in animals and in robots. Steady-state locomotor performance can be assessed using measures such as speed of locomotion normalized by body length, the cost of transport, the Froude number, and the Stroudhal number (24). But the concept of agility still has to be properly defined and quantified. For instance it would be very useful to define normalized agility scores that assess and compare how well an animal or a robot turns, accelerates, jumps, stands-up, etc. A useful attempt to quantify versatility for robots (defined as the ability to cross different types of terrains) can be found in (105). This represents an exciting opportunity for roboticists and biologists to define various agility metrics together, not only for assessing existing animals and robots, but also for setting targets for the design of the next generations of biorobots.

\section{References and Notes:}

1. G. V. Lauder, E. J. Anderson, J. Tangorra, P. G. A. Madden, Fish biorobotics: kinematics and hydrodynamics of self-propulsion. J. Exp. Biol.. 210, 2767-2780 (2007).

2. S. Sefati et al., Mutually opposing forces during locomotion can eliminate the tradeoff between maneuverability and stability. Proc. Natl. Acad. Sci., 201309300 (2013).

3. M. Porez, F. Boyer, A. Ijspeert, others, Improved Lighthill fish swimming model for bio-inspired robotsModelling, computational aspects and experimental comparisons. Int. J. Robot. Res., 1-34 (2014).

4. J. H. Long, Biomimetic robotics: self-propelled physical models test hypotheses about the mechanics and evolution of swimming vertebrates. Proc. Inst. Mech. Eng. Part C-J. Mech. Eng. Sci. - PROC INST MECH ENG C-J MECH E. 221 (2007), doi:10.1243/09544062JMES511.

5. S. Hirose, Biologically Inspired Robots: Snake-like Locomotors and Manipulators (Oxford University Press, Oxford; New York, 1993).

6. R. D. Maladen, Y. Ding, P. B. Umbanhowar, A. Kamor, D. I. Goldman, Mechanical models of sandfish locomotion reveal principles of high performance subsurface sand-swimming. J. R. Soc. Interface. 8, 13321345 (2011).

7. M. Tesch et al., Parameterized and Scripted Gaits for Modular Snake Robots. Adv. Robot.. 23, 1131-1158 (2009).

8. S. Seok et al., in IEEE International Conference on Robotics and Automation (ICRA 2013) (2013), pp. 33073312. 
9. H. Kimura, Y. Fukuoka, A. H. Cohen, Adaptive Dynamic Walking of a Quadruped Robot on Natural Ground Based on Biological Concepts. Int. J. Robot. Res.. 26, 475-490 (2007).

10. A. Spröwitz et al., Towards dynamic trot gait locomotion: Design, control, and experiments with Cheetah-cub, a compliant quadruped robot. Int. J. Robot. Res.. 32, 932-950 (2013).

11. C. Semini et al., in Proceedings of the Institution of Mechanical Engineers, Part I: Journal of Systems and Control Engineering (2011).

12. X. Zhou, S. Bi, A survey of bio-inspired compliant legged robot designs. Bioinspir. Biomim.. 7, 041001 (2012).

13. S. H. Collins, M. Wisse, A. Ruina, A Three-Dimensional Passive-Dynamic Walking Robot with Two Legs and Knees. Int. J. Robot. Res.. 20, 607-615 (2001).

14. T. McGeer, Passive Dynamic Walking. Int. J. Robot. Res.. 9, 62-82 (1990).

15. S. Kajita, B. Espiau, in Handbook of Robotics (Springer, Berlin, 2008; http://link.springer.com/10.1007/978-3540-30301-5_17), pp. 361-389.

16. B. Webb, Can robots make good models of biological behaviour? Behav. Brain Sci.. 24, 1033-1050 (2001).

17. B. Webb, Robots in invertebrate neuroscience. Nature. 417, 359-363 (2002).

18. A. Ananthanarayanan, M. Azadi, S. Kim, Towards a bio-inspired leg design for high-speed running. Bioinspir. Biomim. 7, 046005 (2012).

19. A. J. Ijspeert, A. Crespi, D. Ryczko, J.-M. Cabelguen, From Swimming to Walking with a Salamander Robot Driven by a Spinal Cord Model. Science. 315, 1416-1420 (2007).

20. M. F. Eilenberg, H. Geyer, H. Herr, Control of a powered ankle-foot prosthesis based on a neuromuscular model. IEEE Trans. Neural Syst. Rehabil. Eng. Publ. IEEE Eng. Med. Biol. Soc.. 18, 164-173 (2010).

21. H. M. Herr, A. M. Grabowski, Bionic ankle-foot prosthesis normalizes walking gait for persons with leg amputation. Proc. R. Soc. B Biol. Sci., 457-64 (2011).

22. C. Li, T. Zhang, D. I. Goldman, A Terradynamics of Legged Locomotion on Granular Media. Science. 339, 1408-1412 (2013).

23. M. H. Dickinson et al., How Animals Move: An Integrative View. Science. 288, 100-106 (2000).

24. R. M. Alexander, Principles of Animal Locomotion (Princeton University Press, 2003).

25. P. Holmes, R. Full, D. Koditschek, J. Guckenheimer, The Dynamics of Legged Locomotion: Models, Analyses, and Challenges. SIAM Rev.. 48, 207-304 (2006).

26. R. Pfeifer, J. Bongard, S. Grand, How the body shapes the way we think: a new view of intelligence (MIT press, 2007).

27. R. Pfeifer, M. Lungarella, F. Iida, Self-Organization, Embodiment, and Biologically Inspired Robotics. Science. 318, 1088-1093 (2007).

28. M. Sitti, A. Menciassi, A. Ijspeert, K. H. Low, S. Kim, Survey and Introduction to the Focused Section on BioInspired Mechatronics. IEEEASME Trans. Mechatron.. 18, 409-418 (2013). 
29. A. J. Ijspeert, Central pattern generators for locomotion control in animals and robots: A review. Neural Netw.. 21, 642-653 (2008).

30. D. N. Beal, F. S. Hover, M. S. Triantafyllou, J. C. Liao, G. V. Lauder, Passive propulsion in vortex wakes. J. Fluid Mech.. 549, 385-402 (2006).

31. F. E. Fish, The myth and reality of Gray's paradox: implication of dolphin drag reduction for technology. Bioinspir. Biomim. 1, R17-25 (2006).

32. M. S. Triantafyllou, G. S. Triantafyllou, An efficient swimming machine. Sci. Am.. 272, 64-71 (1995).

33. M. Sfakiotakis, D. M. Lane, J. B. C. Davies, Review of fish swimming modes for aquatic locomotion. IEEE J. Ocean. Eng.. 24, 237-252 (1999).

34. V. van Ginneken et al., Eel migration to the Sargasso: remarkably high swimming efficiency and low energy costs. J. Exp. Biol.. 208, 1329-1335 (2005).

35. M. S. Triantafyllou, G. S. Triantafyllou, D. K. P. Yue, Hydrodynamics of Fishlike Swimming. Annu. Rev. Fluid Mech.. 32, 33-53 (2000).

36. J. M. Anderson, N. K. Chhabra, Maneuvering and Stability Performance of a Robotic Tuna. Integr. Comp. Biol.. 42, 118-126 (2002).

37. J. Liu, H. Hu, Biological Inspiration: From Carangiform Fish to Multi-Joint Robotic Fish. J. Bionic Eng.. 7, 3548 (2010).

38. N. Kato, Control performance in the horizontal plane of a fish robot with mechanical pectoral fins. IEEE J. Ocean. Eng.. 25, 121-129 (2000).

39. C. Stefanini et al., A novel autonomous, bioinspired swimming robot developed by neuroscientists and bioengineers. Bioinspir. Biomim.. 7, 025001 (2012).

40. M. C. Leftwich, E. D. Tytell, A. H. Cohen, A. J. Smits, Wake structures behind a swimming robotic lamprey with a passively flexible tail. J. Exp. Biol.. 215, 416-425 (2012).

41. R. Mittal, H. Dong, M. Bozkurttas, G. Lauder, P. Madden, Locomotion with flexible propulsors: II. Computational modeling of pectoral fin swimming in sunfish. Bioinspir. Biomim.. 1, S35 (2006).

42. I. Akhtar, R. Mittal, G. V. Lauder, E. Drucker, Hydrodynamics of a biologically inspired tandem flapping foil configuration. Theor. Comput. Fluid Dyn.. 21, 155-170 (2007).

43. E. Feron, E. N. Johnson, in Handbook of Robotics (Springer, Berlin, 2008; http://link.springer.com/10.1007/978-3-540-30301-5_45), pp. 1009-1029.

44. B. M. Kulfan, A. J. Colozza, Biomimetics and Flying Technology. Biomim. Nat.-Based Innov. Ed. Bar-Cohen CRC Press Taylor Francis Group Boca Raton FL, 525-674 (2011).

45. R. J. Wood, The First Takeoff of a Biologically Inspired At-Scale Robotic Insect. IEEE Trans. Robot.. 24, 341347 (2008).

46. K. Y. Ma, P. Chirarattananon, S. B. Fuller, R. J. Wood, Controlled Flight of a Biologically Inspired, InsectScale Robot. Science. 340, 603-607 (2013).

47. L. Hines, D. Campolo, M. Sitti, Liftoff of a Motor-Driven, Flapping-Wing Microaerial Vehicle Capable of Resonance. IEEE Trans. Robot.. 30, 220-232 (2014). 
48. J. D. DeLaurier, The development and testing of a full-scale piloted ornithopter. Can. Aeronaut. Space J.. 45, 72-82 (1999).

49. C. P. Ellington, The Aerodynamics of Hovering Insect Flight. I. The Quasi-Steady Analysis. Philos. Trans. R. Soc. Lond. B. Biol. Sci.. 305, 1-15 (1984).

50. M. H. Dickinson, F.-O. Lehmann, S. P. Sane, Wing Rotation and the Aerodynamic Basis of Insect Flight. Science. 284, 1954-1960 (1999).

51. S. N. Fry, R. Sayaman, M. H. Dickinson, The Aerodynamics of Free-Flight Maneuvers in Drosophila. Science. 300, 495-498 (2003).

52. M. J. Elzinga, F. van Breugel, M. H. Dickinson, Strategies for the stabilization of longitudinal forward flapping flight revealed using a dynamically-scaled robotic fly. Bioinspir. Biomim.. 9, 025001 (2014).

53. R. D. Maladen, Y. Ding, C. Li, D. I. Goldman, Undulatory Swimming in Sand: Subsurface Locomotion of the Sandfish Lizard. Science. 325, 314-318 (2009).

54. U. Saranli, M. Buehler, D. E. Koditschek, RHex: A Simple and Highly Mobile Hexapod Robot. Int. J. Robot. Res.. 20, 616-631 (2001).

55. C. Li, S. T. Hsieh, D. I. Goldman, Multi-functional foot use during running in the zebra-tailed lizard (Callisaurus draconoides). J. Exp. Biol.. 215, 3293-3308 (2012).

56. S. Kim et al., Smooth Vertical Surface Climbing With Directional Adhesion. IEEE Trans. Robot.. 24, 65-74 (2008).

57. O. Unver, A. Uneri, A. Aydemir, M. Sitti, in IEEE International Conference on Robotics and Automation (ICRA 2006) (2006), pp. 2329-2335.

58. M. Carlo, S. Metin, A Biomimetic Climbing Robot Based on the Gecko. J. Bionic Eng.. 3, 115-125 (2006).

59. M. J. Spenko et al., Biologically inspired climbing with a hexapedal robot. J. Field Robot.. 25, 223-242 (2008).

60. G. A. Lynch, J. E. Clark, P.-C. Lin, D. E. Koditschek, A bioinspired dynamical vertical climbing robot. Int. J. Robot. Res.. 31, 974-996 (2012).

61. K. Autumn et al., Dynamics of geckos running vertically. J. Exp. Biol.. 209, 260-272 (2006).

62. M. Hutter et al., in 15th International Conference on Climbing and Walking Robot-CLAWAR 2012 (2012; http://infoscience.epfl.ch/record/181042).

63. B. Klaassen, R. Linnemann, D. Spenneberg, F. Kirchner, Biomimetic walking robot SCORPION: Control and modeling. Robot. Auton. Syst.. 41, 69-76 (2002).

64. K. E. Rudman, R. M. Aspden, J. R. Meakin, Compression or tension? The stress distribution in the proximal femur. Biomed. Eng. OnLine. 5, 12 (2006).

65. T. M. Kubow, R. J. Full, The role of the mechanical system in control: a hypothesis of self-stabilization in hexapedal runners. Philos. Trans. R. Soc. Lond. B. Biol. Sci.. 354, 849-861 (1999).

66. J.-M. Cabelguen, C. Bourcier-Lucas, R. Dubuc, Bimodal locomotion elicited by electrical stimulation of the midbrain in the salamander Notophthalmus viridescens. J. Neurosci.. 23, 2434-2439 (2003). 
67. A. Crespi, K. Karakasiliotis, A. Guignard, A. J. Ijspeert, Salamandra Robotica II: An Amphibious Robot to Study Salamander-Like Swimming and Walking Gaits. IEEE Trans. Robot.. 29, 308-320 (2013).

68. D. Owaki, T. Kano, K. Nagasawa, A. Tero, A. Ishiguro, Simple robot suggests physical interlimb communication is essential for quadruped walking. J. R. Soc. Interface. 10 (2013), doi:10.1098/rsif.2012.0669.

69. H. Cruse, What mechanisms coordinate leg movement in walking arthropods? Trends Neurosci.. 13, 15-21 (1990).

70. B. Verrelst et al., The Pneumatic Biped "Lucy" Actuated with Pleated Pneumatic Artificial Muscles. Auton. Robots. 18, 201-213 (2005).

71. F. C. Anderson, M. G. Pandy, Individual muscle contributions to support in normal walking. Gait Posture. 17, 159-169 (2003).

72. A. D. Kuo, Energetics of Actively Powered Locomotion Using the Simplest Walking Model. J. Biomech. Eng.. 124, 113-120 (2001).

73. D. Renjewski, A. Seyfarth, Robots in human biomechanics — a study on ankle push-off in walking. Bioinspir. Biomim.. 7, 036005 (2012).

74. S. Collins, A. Ruina, R. Tedrake, M. Wisse, Efficient Bipedal Robots Based on Passive-Dynamic Walkers. Science. 307, 1082-1085 (2005).

75. P. A. Bhounsule et al., Low-bandwidth reflex-based control for lower power walking: $65 \mathrm{~km}$ on a single battery charge. Int. J. Robot. Res., 0278364914527485 (2014).

76. P. Manoonpong, T. Geng, T. Kulvicius, B. Porr, F. Wörgötter, Adaptive, Fast Walking in a Biped Robot under Neuronal Control and Learning. PLoS Comput Biol. 3, e134 (2007).

77. G. Hirzinger, A. Albu-Schaffer, M. Hahnle, I. Schaefer, N. Sporer, in IEEE International Conference on Robotics and Automation (ICRA 2001) (IEEE, 2001; http://ieeexplore.ieee.org/xpls/abs_all.jsp?arnumber=933136), vol. 4, pp. 3356-3363.

78. L. Sentis, O. Khatib, Synthesis of whole-body behaviors through hierarchical control of behavioral primitives. Int. J. Humanoid Robot.. 02, 505-518 (2005).

79. L. Righetti, J. Buchli, M. Mistry, S. Schaal, in IEEE International Conference on Robotics and Automation (ICRA 2011) (IEEE, 2011; http://ieeexplore.ieee.org/xpls/abs_all.jsp?arnumber=5980156), pp. 1085-1090.

80. N. G. Tsagarakis, S. Morfey, G. M. Cerda, L. Zhibin, D. G. Caldwell, in 2013 IEEE International Conference on Robotics and Automation (ICRA) (2013), pp. 673-678.

81. S. Faraji, S. Pouya, A. Ijspeert, in Robotics Science and Systems (RSS) (2014; http://www.roboticsproceedings.org/rss10/p28.html).

82. C. Semini, V. Barasuol, T. Boaventura, M. Frigerio, J. Buchli, in International Symposium of Robotics Research (ISRR) (2013; http://e-citations.ethbib.ethz.ch/view/pub:124391).

83. D. P. Ferris, G. S. Sawicki, M. A. Daley, A physiologist's perspective on robotic exoskeletons for human locomotion. Int. J. Humanoid Robot. . 04, 507-528 (2007).

84. A. B. Zoss, H. Kazerooni, A. Chu, Biomechanical design of the Berkeley lower extremity exoskeleton (BLEEX). Mechatron. IEEEASME Trans. On. 11, 128-138 (2006). 
85. H. Kawamoto, S. Lee, S. Kanbe, Y. Sankai, in Systems, Man and Cybernetics, 2003. IEEE International Conference on (IEEE, 2003; http://ieeexplore.ieee.org/xpls/abs_all.jsp?arnumber=1244649), vol. 2, pp. 1648 1653.

86. C. J. Walsh, K. Endo, H. Herr, A quasi-passive leg exoskeleton for load-carrying augmentation. Int. J. Humanoid Robot.. 4, 487-506 (2007).

87. J. Gancet et al., in Biomedical Robotics and Biomechatronics (BioRob), 2012 4th IEEE RAS \& EMBS International Conference on (IEEE, 2012; http://ieeexplore.ieee.org/xpls/abs_all.jsp?arnumber=6290688), pp. 1794-1800.

88. J. E. Pratt, B. T. Krupp, C. J. Morse, S. H. Collins, in IEEE International Conference on Robotics and Automation (ICRA 2004) (IEEE, 2004; http://ieeexplore.ieee.org/xpls/abs_all.jsp?arnumber=1307425), vol. 3 , pp. 2430-2435.

89. A. Dollar, H. Herr, Lower Extremity Exoskeletons and Active Orthoses: Challenges and State-of-the-Art. IEEE Trans. Robot.. 24, 144-158 (2008).

90. A. J. Van den Bogert, Exotendons for assistance of human locomotion. Biomed. Eng. Online. 2, 1-8 (2003).

91. J. M. Donelan et al., Biomechanical Energy Harvesting: Generating Electricity During Walking with Minimal User Effort. Science. 319, 807-810 (2008).

92. J. . Paradiso, T. Starner, Energy scavenging for mobile and wireless electronics. IEEE Pervasive Comput.. 4, $18-27$ (2005).

93. L. C. Rome, L. Flynn, E. M. Goldman, T. D. Yoo, Generating electricity while walking with loads. Science. 309, 1725-1728 (2005).

94. H. Geyer, H. Herr, A Muscle-Reflex Model That Encodes Principles of Legged Mechanics Produces Human Walking Dynamics and Muscle Activities. IEEE Trans. Neural Syst. Rehabil. Eng.. 18, 263-273 (2010).

95. J. Markowitz et al., Speed adaptation in a powered transtibial prosthesis controlled with a neuromuscular model. Philos. Trans. R. Soc. Lond. B. Biol. Sci.. 366, 1621-1631 (2011).

96. R. T. Schroer, M. J. Boggess, R. J. Bachmann, R. D. Quinn, R. E. Ritzmann, in IEEE International Conference on Robotics and Automation (ICRA 2004) (2004), vol. 4, pp. 3288-3293 Vol.4.

97. T. Libby et al., Tail-assisted pitch control in lizards, robots and dinosaurs. Nature. 481, 181-184 (2012).

98. R. J. Full, D. E. Koditschek, Templates and anchors: neuromechanical hypotheses of legged locomotion on land. J. Exp. Biol.. 202, 3325-3332 (1999).

99. R. Blickhan, R. J. Full, Similarity in multilegged locomotion: bouncing like a monopode. J. Comp. Physiol. A. 173, 509-517 (1993).

100. B. Vanderborght et al., Variable impedance actuators: A review. Robot. Auton. Syst.. 61, 1601-1614 (2013).

101. S. Kim, C. Laschi, B. Trimmer, Soft robotics: a bioinspired evolution in robotics. Trends Biotechnol.. 31, 287-294 (2013).

102. M. T. Tolley et al., A Resilient, Untethered Soft Robot. Soft Robot. (2014), doi:10.1089/soro.2014.0008. 
103. R. J. Lock, S. C. Burgess, R. Vaidyanathan, Multi-modal locomotion: from animal to application. Bioinspir. Biomim. 9, 011001 (2014).

104. C. Georgiades et al., in IEEE/RSJ International Conference on Intelligent Robots and Systems (IROS 2004) (2004), vol. 4, pp. 3525-3531 vol.4.

105. C. Nie, X. Pacheco Corcho, M. Spenko, Robots on the Move: Versatility and Complexity in Mobile Robot Locomotion. IEEE Robot. Autom. Mag.. 20, 72-82 (2013).

Acknowledgments: I thank M. Ajallooeian, J. Buchli, P. Eckert, N. van der Noot, and D. Renjewski for useful comments on the manuscript. Supported by the Swiss National Center of Competence in Research in Robotics, the Swiss National Science Foundation, the Swiss NanoTera program, the EU-funded WalkMan project (FP7-ICT 611832) and the EU-funded Symbitron project (FP7-ICT 661626). 